\title{
Prognostic significance of NDRG2 combined with EGFR-sensitizing mutations in lung adenocarcinoma
}

Bo Yang ( $\nabla$ yangbochest@126.com )

Tianjin First Central Hospital https://orcid.org/0000-0003-0052-048X

Linlin Ji

Tianjin Medical University

Yiling Feng

Armed Police Force

Xiao-Ping Li

Tianjin First Central Hospital

Hong-Gang Zhou

Nankai University

Tao Jiang

Xi'an Tangdu Hospital of No4 Military Medical University

Ting Xiao

Nankai University

Xiao-He Li

Nankai University

Liang Zhang

Tianjin First Central Hospital

Hongliang Xu

Tianjin Baodi Hospital

Guang-Shun Wang

Tianjin Baodi Hospital

Wei-Dong Zhang

Tianjin First Central Hospital

Research article

Keywords: N-myc downstream-regulated gene 2, Epidermal growth factor receptor, Lung adenocarcinoma, Prognosis

Posted Date: August 26th, 2020 
DOI: https://doi.org/10.21203/rs.2.14181/v5

License: (c) (1) This work is licensed under a Creative Commons Attribution 4.0 International License. Read Full License 


\section{Abstract}

Background: N-myc downstream-regulated gene 2 (NDRG2) plays a substantial role in lung adenocarcinoma (LUAD). Epidermal growth factor receptor (EGFR)-sensitizing mutation could significantly improve prognosis in patients with LUAD. Here, we aimed to elucidate the prognostic value of NDRG2 combined with EGFR-sensitizing mutations in patients with LUAD.

Methods: Lung parenchyma specimens obtained during surgery or CT-guide percutaneous lung puncture biopsy for the NDRG2 protein and EGFR genomic testing were obtained. Associations between NDRG2/EGFR and clinicopathological characteristics of patients with LUAD were extracted from the Tianjin First Central Hospital in China between June 2013 and June 2014.

Results: The expression of NDRG2 was significantly decreased in patients with LUAD. Expressions of NDRG2 and EGFR-sensitizing mutations showed positive correlations with survival. ROC curve analyses showed that the diagnostic value of NDRG2 combined with EGFR in LUAD was significantly higher than that of each biomarker alone. Expression of NDRG2 and EGFR-sensitizing mutations were associated with the longer overall survival (OS), disease-free survival (DFS) and progression-free survival (PFS). Advanced stages were significantly associated with low expression of NDRG2. In multivariate analysis, compared with other patients, NDRG2 (+)/EGFR (+) was independently associated with prolonged OS and PFS.

Conclusion: NDRG2 combined with EGFR-sensitizing mutations might be valuable markers to evaluate the prognosis of LUAD patients.

\section{Background}

Lung cancer remains the leading cause of cancer-related death worldwide, accounting for $19.4 \%$ of cancer mortality among adults [1]. Among lung cancers, small-cell lung cancers represent approximately $15 \%$ of the cases and non-small cell lung cancers (NSCLCs) approximately $85 \%$. NSCLC includes lung adenocarcinoma (LUAD), which is the most prevalent subtype of lung cancer [2]. Only approximately $20 \%$ of patients with NSCLC can be potentially treated by resection, and the remaining are diagnosed in advanced stages [3]. Despite advances accomplished in terms of early detection and standard treatments such as surgery, chemotherapy, radiotherapy, and iodine-125 (125I) brachytherapy, the overall survival (OS) of NSCLC still remains poor $[3,4]$.

Targeted therapy, such as tyrosine kinase inhibitors (TKIs), has recently emerged as a new therapeutic approach for patients with advanced NSCLC, especially for LUAD harboring EGFR mutations, showing better progression-free survival (PFS) compared with wild type tumors $[5,6]$. Nevertheless, after surgical resection, several patients eventually relapse due to inevitable drug resistance, and patients lacking driver oncogene aberrations are still treated with traditional regimens, with poor outcomes [7]. Hidayat et al. found that FBXW7 expression in CD133-positive cells was increased, and c-MYC expression was decreased in gefitinib-resistant tumors from PC9 cells in mice and in nine out of 14 tumor specimens 
from patients with EGFR-mutant NSCLC and acquired resistance to gefitinib [8]. Another study reported that hyperprogressive disease (HPD) promptly leads to death in patients harboring EGFR exon 20 insertion mutation and MYC amplification [9]. Consequently, the interaction between MYC and EGFR mutations may play a significant role in malignant tumor cells, involving cell proliferation, invasion, and metastasis.

The N-myc downstream-regulated gene (NDRG) family consists of four members: NDRG1, NDRG2, NDRG3, and NDRG4 [10]. A previous study showed that NDRG2 exerts important functions in cell differentiation and tumor suppression. Researches revealed that DNA damage, hypoxia, and glucocorticoids promoted NDRG2 expression, and NDRG2 can be transcriptionally activated by p53 and HIF1-a [11]. Decreased expression of NDRG2 has been found in several types of human cancers, such as lung cancer, bladder cancer, colon cancer, pancreatic cancer, thyroid cancer, glioblastoma, melanoma, and meningioma; besides, NDRG2 was found as a candidate tumor suppressor gene [12]. Although a previous analysis revealed that NDRG2 might serve as a novel prognostic marker in human lung cancer, its prognostic value among patients with mutant EGFR adenocarcinoma has not been studied adequately.

Accordingly, the purpose of this study was to explore the prognostic impact of NDRG2 expression based on the EGFR-sensitizing mutation status in LUAD patients.

\section{Methods}

Patients

A total of 89 LUAD patients were prospectively enrolled in this cohort study at the Tianjin First Central Hospital between June 2013 and June 2014. Patients who received neoadjuvant chemotherapy, radiotherapy, or those diagnosed with distant metastasis at initial presentation were excluded. Clinicopathological characteristics including survival data were retrospectively collected by review of medical records (Table 1).

Tumor staging and grading were according to the 8th edition of the Union for International Cancer Control (UICC) TNM classification of malignant tumors. Hematoxylin and eosin (H\&E) staining of tissue slides was performed and verified by two board-certified pathologists.

Among the patients (89), 34 cases underwent curative-intent surgery (normal tissues were removed from at least $5 \mathrm{~cm}$ away from the edge of the tumors); 55 cases with advanced LUAD were treated with iodine$125\left({ }^{125}\right)$ brachytherapy.

All tumor samples obtained during surgery or CT-guide percutaneous lung puncture biopsy underwent genomic testing to detect EGFR mutations.

EGFR mutation analysis 
DNA from formalin-fixed, paraffin-embedded tumor tissue and matched blood samples was extracted. Comprehensive genomic profiling was performed by next generation sequencing (NGS) with a 37 or 450 cancer related gene panel covering the whole EGFR gene at a mean coverage depth of $>800 \mathrm{x}$. The genomic alterations including single base substitution and deletions were assessed. The EGFR (-) denote wild-type, while EGFR (+) denote mutation.

Immunohistochemistry

In the present study, formalin-fixed paraffin-embedded tissue sections (thickness, 4- $\mu \mathrm{m}$ ) were used for detecting the expression of NDRG2. Tissue sections were dewaxed, rehydrated, antigen-retrieved, and cooled to room temperature. The sections were incubated with mouse monoclonal anti-NDRG2 antibody (1:500 dilution, Santa Cruz Biotechnology, Santa Cruz, CA, USA) at $4^{\circ} \mathrm{C}$ overnight, rinsed with phosphatebuffered saline (PBS), and incubated with horseradish peroxidase (HRP)-labeled goat anti-mouse secondary antibody for $60 \mathrm{~min}$. NDRG2 expression was revealed using 3,3'-diaminobenzidine (DAB) as the chromogen. Negative control was performed by replacing the primary antibody with normal mouse serum. The brown or yellow staining was identified as a positive expression. The total staining score of $0-$ 12 was considered in a semi-quantitative manner and stratified as follows: negative (-, range of score: 01), weak (+, range of score: $2-4)$, moderate ( ++ , range of score: $5-8)$, or strong (+++, range of score: 9-12). The tumor specimens were divided into the NDRG2 (-) group (range of score: 0-4) and the NDRG2 (+) group (range of score: 5-12) [12].

Western blot analysis

Total protein concentration was measured by the bicinchoninic acid (BCA) assay kit. The proteins were separated by $10 \%$ sodium dodecyl sulfate-polyacrylamide gel electrophoresis (SDS-PAGE) and transferred onto polyvinylidene fluoride (PVDF) membranes. The membranes were incubated with mouse anti-human NDRG2 antibody (1:500, Santa Cruz Biotechnology, Santa Cruz, CA, USA) and GAPDH antibody (1:1000, Beyotime Institute of Biotechnology, Haimen, China) at $4^{\circ} \mathrm{C}$ overnight after being blocked with $5 \%$ non-fat milk for $1 \mathrm{~h}$. The membranes were washed and incubated with HRP-conjugated secondary antibody (Santa Cruz Biotechnology, Santa Cruz, CA, USA). The blots were visualized using an enhanced chemiluminescence kit (Amersham Pharmacia Biotech, Arlington Heights, IL, USA) according to the manufacturer's instructions. Each experiment was performed in triplicate.

\section{qRT-PCRanalysis}

The total RNA was extracted from the fresh tissues using TRIzol reagent (Invitrogen, Carlsbad, CA, USA) according to the manufacturer's instructions. The first chain of cDNA was synthesized by reverse transcription with TaqMan ${ }^{\circledR}$ Reverse Transcription Reagents (Applied Biosystems, Grand Island, NY). GAPDH was used as internal control. The sequences of the primers were: NDRG2, forward-5'-ATG GCG GAG CTG CAG GAG GTC-3', and reverse-5'-AAC AAG GGC CAT TCA ACA GGA GAC-3'; GAPDH, forward-5'GCC TCA AGA TCA GCA AT-3' and reverse-5'-AGG TCC ACC ACT GAC ACG TT-3'. RT-PCR reaction was 
performed using the CFX96 Touch PCR system (Bio-Rad). The relative mRNA expression of NDRG2 was calculated by the $-2^{\Delta \Delta \mathrm{Ct}}$ method. Each experiment was undertaken in triplicate.

Statistical analysis

Statistical analysis was performed with SPSS software (version 18.0; SPSS, Chicago, IL, USA). Continuous data were presented as means \pm standard deviations (SD). Differences between categorical variables were investigated by the chi-square test. The differences in means between groups were analyzed using the Student's t-test. The relationship between NDRG2 expression levels and clinicopathological factors was analyzed using the Wilcoxon-Mann-Whitney test. The diagnostic accuracy of NDRG2 and EGFR for LUAD was evaluated using receiver operating characteristic (ROC) curves.

The patient's survival curves were evaluated using the Kaplan-Meier method, and PFS, DFS and OS were compared using the log-rank test as well as univariate Cox proportional-hazards regression model. To adjust for the clinically important factors, multivariate Cox proportional-hazards regression model was utilized. All statistical tests were two-sided, and $\mathrm{P}<0.05$ was considered statistically significant.

\section{Results}

\section{Clinical characteristics of the patients}

In total, 89 patients with lung adenocarcinoma were included in this retrospective study. The median age of the study population was 65.6 years old (range, 38-86) and $52(58.4 \%)$ were male. NDRG2 expression was positive in $36(40.4 \%)$ patients. EGFR mutations (denoted as EGFR (+)) were detected in 29 (32.6\%) patients. 69 (77.5\%) patients received postoperative adjuvant chemotherapy. The baseline characteristics of the study population and their associations with NDRG2 and EGFR are summarized in Table 1.

\section{Driver mutations in the LUAD}

The EGFR mutation patterns are shown in Table 2. The most frequent mutations found in EGFR in turn were the exon 19 deletion (51.7\%), followed by the activation-mutation of exon 21 (L858R) $(41.4 \%$ of the cases).

\section{Expression of NDRG2 and EGFR mutations correlates with clinicopathological characteristics of LUAD}

The expression and distribution of NDRG 2 in 34 cases of lung adenocarcinoma were examined by IHC (Figure $1 \mathrm{~A}-\mathrm{C}$ ). In total, $23(67.6 \%$ ) cases were highly positive for NDRG2 (denoted as NDRG2 (+)) while $11(32.4 \%)$ cases were negative-to-weakly positive (denoted as NDRG2 (-)). 34 cases of lung adenocarcinoma were also examined by Western blotting and qRT-PCR revealed that the expression of NDRG2 at the protein (Figure 1 D) and mRNA (Figure $1 \mathrm{E}$ ) levels in LUAD was significantly lower compared with adjacent non-carcinoma tissues. 
To evaluate the expression of NDRG2 in different stages of LUAD, we divided LUAD samples into four groups according to the disease stage. The expression of NDRG2 decreased as the TNM stage increases (Figure $1 \mathrm{~F}-\mathrm{G}$ ).

Statistically significant correlations were found between the level of NDRG2 expression and the presence of vascular invasion $(P<0.001)$, and TNM stage $(P<0.001)$. Meanwhile, statistically significant correlations were found between the wild-type of EGFR (denoted as EGFR (-)) and the poor degree of histological differentiation $(P=0.001)$, the presence of vascular invasion $(P<0.001)$, TNM stage $(P<$ $0.001)$ and smoking history $(P=0.003)($ Table 1$)$.

\section{The diagnostic value of the combination of NDRG2 and EGFR in LUAD}

The ROC curves for the analyses of NDRG2 and EGFR in LUAD were plotted. The AUCs were as follows: $0.843(95 \% \mathrm{Cl} 0.753-0.933, \mathrm{p}<0.001)$ for NDRG2 and 0.735 (95\% Cl 0.613-0.857, $\mathrm{p}=0.001)$ for EGFR. When NDRG2 and EGFR were combined, the AUC was 0.909 (95\% $\mathrm{Cl} 0.839-0.979, \mathrm{p}<0.001$; Figure 2).

\section{Prognostic significance of NDRG2 and EGFR}

The Kaplan-Meier analysis for postoperative OS and DFS showed that patients with preserved NDRG2 and mutant EGFR had longer OS, and better DFS than patients with reduced NDRG2 and wild-type EGFR (Figure 3). The results also showed that iodine-125 radioactive seeds brachytherapy for advanced LUAD with low expression level of NDRG2 and wild-type EGFR led to significantly poor OS and PFS (Figure 4).

In subgroup univariate analysis according to the surgery, LUAD patients with reduced NDRG2 expression was significantly associated with a lower OS (hazard ratio (HR) $0.267,95 \%$ confidence interval (Cl) 0.107 0.662 , log-rank $p=0.004)$ and showed a trend toward lower DFS (HR 0.306, 95\% $\mathrm{Cl} 0.101-0.927$, log-rank $\mathrm{p}=0.036)$. EGFR mutation was significantly associated with higher $\mathrm{OS}$ rate $(\mathrm{HR} 0.139,95 \% \mathrm{Cl} 0.045$ 0.428 , log-rank $p=0.001)$ and better DFS (HR 0.169, 95\% Cl 0.046-0.801, log-rank $p=0.007)$ (Table 3).

In subgroup univariate analysis according to the iodine-125, highly positive NDRG2 expression was associated with better OS (hazard ratio (HR) 0.484, 95\% confidence interval (Cl) 0.251-0.935, log-rank $p=$ $0.031)$ and PFS (HR 0.489, 95\% $\mathrm{Cl} 0.251-0.950$, log-rank $\mathrm{p}=0.035)$. EGFR mutation showed a trend toward better OS (HR 0.071, 95\% Cl 0.023-0.217, log-rank $p=0.000)$ and PFS (HR 0.188, $95 \% \mathrm{Cl} 0.082-$ 0.432 , log-rank $p=0.000)($ Table 3$)$.

In multivariate analysis, NDRG2 and EGFR may be two prognostic factors for OS of LUAD patients (Table 4).

\section{Prognostic significance of the combinations of NDRG2 and EGFR}

The patients into two groups in terms of the marker combinations as follows: the first group consisted of patients who were NDRG2 (+)/EGFR (+), and the others group consisted of patients who were NDRG2 $(+) / \operatorname{EGFR}(-), \operatorname{NDRG} 2(-) / \operatorname{EGFR}(+)$, and NDRG2 (-)/EGFR (-). 
Kaplan-Meier analysis (Figure 5) showed that OS was lower in the others group than in the NDRG2 $(+) /$ EGFR (+) group; the DFS and PFS were also significantly poorer in the former.

In univariate analysis according to the surgery, NDRG2 (+)/EGFR (+) was associated with both longer OS (HR 0.145, 95\% Cl 0.042-0.504, log-rank $\mathrm{p}=0.002$ ) and longer DFS (HR 0.219, 95\% Cl 0.060-0.801, logrank $p=0.022$ ) (Table 3). In multivariate analysis according to the surgery, NDRG2 (+)/EGFR (+) was significantly associated with a longer OS (HR $0.154,95 \% \mathrm{Cl} 0.030-0.795, \mathrm{p}=0.025)$ but not with DFS (HR $0.241,95 \%$ Cl 0.037-1.566, $p=0.244$ ) (Table 4).

In univariate analysis according to the iodine-125, NDRG2 (+)/EGFR (+) group showed better OS (HR $0.269,95 \% \mathrm{Cl} 0.104-0.696, \mathrm{p}=0.007)$ and PFS (HR 0.272, 95\% Cl 0.103-0.716, p = 0.008) (Table 3). In multivariate analysis according to the iodine-125, NDRG2 (+)/EGFR (+) group showed better OS (HR $0.144,95 \% \mathrm{Cl} 0.031-0.749, \mathrm{p}=0.018$ ) and PFS (HR 0.399, 95\% Cl 0.086-1.040, $\mathrm{p}=0.048)$. In addition, vascular invasion and TNM stage were also shown to be independent prognostic factors after controlling for all other clinicopathological factors (Table 4).

\section{Discussion}

To improve the prediction of lung cancer survival, several tumor markers have been assessed and extensively used $[13,14]$, but each marker has its own specificity and sensitivity, which might lead to limitations in prognostic ability. The combined detection of tumor markers may be of great importance for improving the prediction of lung cancer survival.

MYC influences growth, proliferation, differentiation, and apoptosis of cancer cells through regulating the expression of numerous genes[15]. In addition, MYC governs events associated with tumor progression, including genetic stability, migration, and angiogenesis [16]. Two human cDNAs, encoding NDRG3 and NDRG4, are homologous to NDRG1. These two genes, together with NDRG1 and a previously deposited cDNA (designated NDRG2), constitute the NDRG gene family, which is identified as a novel type of mycrepressed genes and believed to play important roles in diverse biological processes of human cancer [17]. Previous studies reported that NDRG2 was associated with human lung cancer, and the decreased expression of NDRG2 was correlated with a worse outcome of lung cancer patients $[12,18]$. Similarly, we showed significantly decreased NDRG2 levels in patients with lung adenocarcinoma. NDRG2 levels showed opposite correlated with cancer progression on the basis of the TNM staging system. Advanced disease stage was significantly associated with low NDRG2, suggesting that NDRG2 can be a promising tumor marker of lung adenocarcinoma reflecting systemic tumor burden. We also confirmed that NDRG2 positivity was correlated with EGFR mutations.

EGFR mutations are detectable in approximately $10-35 \%$ of lung adenocarcinoma [19]. Several EGFRtargeting tyrosine kinase inhibitors (TKIs) have demonstrated higher objective response rates (ORR) and PFS over chemotherapy in patients with advanced disease, making first-line EGFR TKIs the treatment of choice for this subtype of adenocarcinoma [20,21,22]. Catherine Labbé et al. demonstrated that patients with dual TP53/EGFR mutations, especially missense mutations, had marginally lower response rates 
and shorter PFS when treated with EGFR TKI therapy [23]. Feng Wang et al. also showed that mutant TP53 is a poor prognostic factor in LUAD patients, and the prognosis of TP53/EGFR co-mutation is worse [24]. They also demonstrated that, there was no significant difference in OS benefit when patients with EGFR mutations were compared with those with EGFR wild type. However, our data did show a significant difference in OS of patients with EGFR mutations. Ji Young Park et al. also noted that patients with EGFRpositive lung adenocarcinoma had longer OS than those with EGFR-negative malignancy [6]. Meanwhile, to show the performance of the prediction mode in LUAD and normal controls, the ROC curves were plotted. The results indicated that the AUC of NDRG2 combined with EGFR levels was 0.909, indicating it might be a potential biomarker for LUAD.

In this current study, we first observed the correlation between NDRG2 and EGFR in LUAD patients and the prediction of LUAD prognosis based on the expression of NDRG2 and mutant EGFR. Our results also showed that NDRG2 expression and EGFR mutation correlated with vascular invasion and TNM stage of LUAD patients. MYC and mutant EGFR have been identified as potential biomarkers that can predict the efficacy of targeted therapy $[25,26,27]$. Our detection showed the possibility of NDRG2 and EGFR as a useful biomarker in the diagnosis or prediction of clinical outcomes for LUAD. In addition, we reclassified the patients into two groups using the marker combinations of NDRG2 and EGFR to further investigate the prognostic impact of NDRG2 and EGFR and found that patients with NDRG2 (+)/EGFR (+) had a significantly better OS and PFS than those with other marker combinations.

This study has several limitations. First, it was a retrospective study performed at a single center. Thus, a wide multicentric study is probably required to confirm our results. Second, the sample size was very limited. The present study, in itself, cannot be used to change clinical practice and to propose the use of a new test. Those results have to be validated using a large sample size. Hence, with increasing numbers, it should be possible to evaluate the true effect of NDRG2/EGFR as both a prognostic and predictive variable.

\section{Conclusions}

In summary, this study reported the different expression levels of NDRG2 in patients with LUAD. In addition, for the first time, the relationship between NDRG2/EGFR and clinicopathological characteristics of patients with LUAD, especially prognosis status, was investigated. NDRG2/EGFR can be used as a novel prognostic biomarker for patients with LUAD.

\section{Declarations}

The authors declare no support from any organizations for the submitted work. The design of the study, the analyses and the writing of the manuscript were solely the responsibility of the authors.

\section{Ethics approval and consent to participate}


This study was conducted in accordance with the Declaration of Helsinki and was confirmed by the Ethics Committee of Tianjin First Central Hospital of Nankai University (Tianjin, China; approval no. 2018N054KY). All patients signed the written informed consent forms.

\section{Consent for publication}

Not applicable

\section{Availability of data and materials}

The data of the current research are available from the corresponding author on a reasonable request.

\section{Competing interests}

The authors declare that there are no conflicts of interest.

\section{Funding}

This study was financially supported by the Science \& Technology Program of Tianjin First Central Hospital, Nankai University (Grant Nos. CM201803 and CF201807) in the design of the study, collection, analysis, and interpretation of data.

\section{Authors' contributions}

BY, GSW, TJ, and WDZ conceived of the study. LJ, HGZ, TX, and XHL performed data analysis for experiments. BY, HGZ, LJ, and FY. drafted the final version of the manuscript and figure legends. BY, XPL, $\mathrm{XHL}$, and $\mathrm{LZ}$ revised the figures, added critical content to the discussion, and were responsible for revising all portions of the submitted portion of the manuscript. TX and HX performed the experiments using lung adenocarcinoma and control tissue. All contributors meet the criteria for authorship. All of the authors read and approved the final manuscript.

\section{Acknowledgments}

We thank Prof. Lei Liu, Lan Wu, Wei Gao, and Wen Hou from the Department of Science and Education of Tianjin First Central Hospital of Nankai University for their kind technical assistance.

\section{Abbreviations}

NDRG2: N-Myc downstream-regulated gene2

LUAD: Lung adenocarcinoma

EGFR: Epidermal growth factor receptor

CEA: Carcinoembryonic antigen 
NSCLC: Non-small cell lung cancer

TKls: Tyrosine kinase inhibitors

HPD: Hyperprogressive disease

HIF1-a: Hypoxia-inducible factor (HIF)1-a

PBS: Phosphate-buffered saline

DAB: 3,3'-Diaminobenzidine

SDS-PAGE: Sodium dodecyl sulfate-polyacrylamide gel electrophoresis

PVDF: Polyvinylidene fluoride

qRT-PCR: Quantitative real-time reverse transcription polymerase chain reaction

HRP: Horseradish peroxidase

NGS: Next generation sequencing

GAPDH: Glyceraldehyde-3-phosphate dehydrogenase

SD: Standard deviation

TNM: Tumor lymph node metastasis

OS: Overall survival

DFS: Disease-free survival

PFS: Progression-free survival

ROC: Receiver operating characteristic

AUC: Area under curve

SE: Standard error

HR: Hazard ratio

Cl: Confidence intervals

\section{References}


1. Yoon HI, Kwon OR, Kang KN, et al. Diagnostic Value of Combining Tumor and Inflammatory Markers in Lung Cancer. J Cancer Prev. 2016;21(3):187-193. doi: 10.15430/JCP.2016.21.3.187.

2. Chalela R, Bellosillo B, Curull V, et al. EGFR and KRAS Mutations in the Non-Tumoral Lung. Prognosis in Patients with Adenocarcinoma. J Clin Med. 2019;8(4):529. doi: 10.3390/jcm8040529.

3. Dhont L, Pintilie M, Kaufman E, et al. Helicase-like transcription factor expression is associated with a poor prognosis in Non-Small-Cell Lung Cancer (NSCLC). BMC cancer 2018, 18(1):429. doi: 10.1186/s12885-018-4215-y.

4. Zhang W, Li J, Li R, et al. Efficacy and safety of iodine-125 radioactive seeds brachytherapy for advanced non-small cell lung cancer-A meta-analysis. Brachytherapy 2018, 17(2):439-448.doi: 10.1016/j.brachy.2017.11.015.

5. Chiu $\mathrm{CH}$, Chou TY, Chiang $\mathrm{CL}$, et al. Should EGFR mutations be tested in advanced lung squamous cell carcinomas to guide frontline treatment? Cancer chemotherapy and pharmacology. 2014;74(4):661665.doi: 10.1007/s00280-014-2536-3.

6. Park JY, Jang SH, Kim HI, et al. Thyroid transcription factor-1 as a prognostic indicator for stage IV lung adenocarcinoma with and without EGFR-sensitizing mutations. BMC cancer 2019, 19(1):574.doi: 10.1186/s12885-019-5792-0.

7. Deng $C$, Zhang $Y, M a Z$, et al. Prognostic value of epidermal growth factor receptor gene mutation in resected lung adenocarcinoma. J Thorac Cardiovasc Surg. 2020;S0022-5223(20)31529-4. doi: 10.1016/j.jtcvs.2020.05.099.

8. Hidayat M, Mitsuishi Y, Takahashi F, et al. Role of FBXW7 in the quiescence of gefitinib-resistant lung cancer stem cells in EGFR-mutant non-small cell lung cancer. Bosn J Basic Med Sci. 2019;19(4):355-367. doi: $10.17305 /$ bjbms.2019.4227.

9. Huang X, Xia L, Lan F, et al. Treatment of Nivolumab Results in Hyperprogressive Disease in a Patient Harboring EGFR Exon 20 Insertion and MYC Amplification. Journal of thoracic oncologyJ Thorac Oncol. 2019;14(9):e189-e191. doi: 10.1016/j.jtho.2019.04.009.

10. Boulkroun S, Fay M, Zennaro MC, et al. Characterization of rat NDRG2 (N-Myc downstream regulated gene 2), a novel early mineralocorticoid-specific induced gene.J Biol Chem. 2002;277(35):31506-31515. doi: 10.1074/jbc.M200272200.

11. Guo Y, Li X, Sun X, et al. Combined Aberrant Expression of NDRG2 and LDHA Predicts Hepatocellular Carcinoma Prognosis and Mediates the Anti-tumor Effect of Gemcitabine. Int J Biol Sci. 2019;15(9):17711786. doi: 10.7150/ijbs.35094. eCollection 2019.

12. Li SJ, Wang WY, Li B, et al. Expression of NDRG2 in human lung cancer and its correlation with prognosis. Med Oncol. 2013;30(1):421. doi: 10.1007/s12032-012-0421-7. 
13. Wang XF, Wu YH, Wang MS, et al. CEA, AFP, CA125, CA153 and CA199 in malignant pleural effusions predict the cause. Asian Pac J Cancer Prev. 2014;15(1):363-8. doi: 10.7314/apjcp.2014.15.1.363.

14. Jiang ZF, Wang M, Xu JL. Thymidine kinase 1 combined with CEA, CYFRA21-1 and NSE improved its diagnostic value for lung cancer. Life Sci. 2018;194:1-6. doi: 10.1016/j.Ifs.2017.12.020.

15. Vervoorts J, Luscher-Firzlaff J, Luscher B. The ins and outs of MYC regulation by posttranslational mechanisms. J Biol Chem. 2006;281(46):34725-34729. doi: 10.1074/jbc.R600017200.

16. Liu J, Levens D. Making myc. Curr Top Microbiol Immunol. 2006;302:1-32. doi: 10.1007/3-540-329528_1.

17. Yang YQ, Tian T, Zhu HY, et al. NDRG2 mRNA levels and miR-28-5p and miR-650 activity in chronic lymphocytic leukemia. BMC Cancer. 2018;18(1):1009. doi: 10.1186/s12885-018-4915-3.

18. Wang $\mathrm{H}$, Wang W, Wang $\mathrm{X}$, et al. Reduced N-Myc downstream-regulated gene 2 expression is associated with CD24 upregulation and poor prognosis in patients with lung adenocarcinoma. Med Oncol. 2012;29(5):3162-3168. doi: 10.1007/s12032-012-0231-y.

19. Cheng L, Alexander RE, Maclennan GT, et al. Molecular pathology of lung cancer: key to personalized medicine. Mod Pathol. 2012;25(3):347-69. doi: 10.1038/modpathol.2011.215.

20. Maemondo M, Inoue A, Kobayashi K, et al. Gefitinib or chemotherapy for non-small-cell lung cancer with mutated EGFR. N Engl J Med. 2010;362(25):2380-2388. doi: 10.1056/NEJMoa0909530.

21. Tran VT, Phan TT, Nguyen ST, et al. Smoking habit and chemo-radiotherapy and/or surgery affect the sensitivity of EGFR plasma test in non-small cell lung cancer. BMC Res Notes. 2020;13(1):367.doi: 10.1186/s13104-020-05209-9.

22. Sun $Y$, Wu M, Zhou M et al. Management of medically inoperable and tyrosine kinase inhibitor-naïve early-stage lung adenocarcinoma with epidermal growth factor receptor mutations: a retrospective multiinstitutional analysis. BMC Cancer. 2020,20(1):646. doi: 10.1186/s12885-020-07122-7.

23. Catherine L , Michael C , Grzegorz JK. et al. Prognostic and predictive effects of TP53 co-mutation in patients with EGFR-mutated non-small cell lung cancer (NSCLC). Lung Cancer . 2017;111:23-29. DOI:

10.1016/j.lungcan.2017.06.014

24. Wang F , Zhao N, Gao G. et al. Prognostic value of TP53 co-mutation status combined with EGFR mutation in patients with lung adenocarcinoma. J Cancer Res Clin Oncol. 2020 Aug 2. doi: $10.1007 / \mathrm{s} 00432-020-03340-5$.

25. Yamashita T, Higashi M, Momose S, et al. Decreased MYC-associated factor X (MAX) expression is a new potential biomarker for adverse prognosis in anaplastic large cell lymphoma. Sci Rep. 2020;10(1):10391. doi: 10.1038/s41598-020-67500-w. 
26. Kwak Y, Yun S, Nam SK, et al. Comparative analysis of the EGFR, HER2, c-MYC, and MET variations in colorectal cancer determined by three different measures: gene copy number gain, amplification status and the 2013 ASCO/CAP guideline criterion for HER2 testing of breast cancer. J Transl Med. 2017;15(1):167. doi: 10.1186/s12967-017-1265-x.

27. Li WY, Zhao TT, Xu HM, et al. The role of EGFR mutation as a prognostic factor in survival after diagnosis of brain metastasis in non-small cell lung cancer: a systematic review and meta-analysis. BMC cancer 2019, 19(1):145.doi: 10.1186/s12885-019-5331-z.

\section{Tables}




\begin{tabular}{|c|c|c|c|c|c|c|c|}
\hline Clinicopatholog-ic Variables & $\begin{array}{l}\text { All } \\
(n=89)\end{array}$ & $\begin{array}{l}\text { NDRG2 (+) } \\
(\mathrm{n}=36)\end{array}$ & $\begin{array}{l}\text { NDRG2 (-) } \\
(\mathrm{n}=53)\end{array}$ & P-value & EGFR $(+)(n=29)$ & $\begin{array}{l}\text { EGFR (-) } \\
(\mathrm{n}=60)\end{array}$ & P-value \\
\hline Gender & & & & 0.988 & & & 0.177 \\
\hline Male & 52 & 21 & 31 & & 14 & 38 & \\
\hline Female & 37 & 15 & 22 & & 15 & 22 & \\
\hline Age (years) & & & & 0.996 & & & 0.887 \\
\hline$<65$ & 42 & 17 & 25 & & 14 & 28 & \\
\hline$\geq 65$ & 47 & 19 & 28 & & 15 & 32 & \\
\hline Smoking status & & & & 0.771 & & & 0.003 \\
\hline No Smoking history & 41 & 14 & 19 & & 20 & 21 & \\
\hline Smoking history & 48 & 22 & 34 & & 9 & 39 & \\
\hline Blood type & & & & 0.265 & & & 0.051 \\
\hline A & 29 & 14 & 15 & & 11 & 18 & \\
\hline B & 27 & 7 & 20 & & 5 & 22 & \\
\hline $\mathrm{AB}$ & 9 & 5 & 4 & & 5 & 4 & \\
\hline $\mathrm{O}$ & 24 & 10 & 14 & & 8 & 16 & \\
\hline Stage & & & & $<0.001$ & & & $<0.001$ \\
\hline I & 25 & 18 & 7 & & 17 & 8 & \\
\hline II & 15 & 9 & 6 & & 7 & 8 & \\
\hline III & 10 & 2 & 8 & & 2 & 8 & \\
\hline IV & 39 & 7 & 32 & & 3 & 36 & \\
\hline Grade & & & & 0.077 & & & 0.001 \\
\hline 1 & 30 & 16 & 14 & & 17 & 13 & \\
\hline $2-3$ & 59 & 20 & 39 & & 12 & 47 & \\
\hline Vascular invasion & & & & $<0.001$ & & & $<0.001$ \\
\hline No & 51 & 19 & 32 & & 27 & 24 & \\
\hline Yes & 38 & 34 & 4 & & 2 & 36 & \\
\hline EGFR-sensitizing mutation & & & & $<0.001$ & & & N/A \\
\hline Wild-type (-) & 60 & 16 & 44 & & $\mathrm{~N} / \mathrm{A}$ & $\mathrm{N} / \mathrm{A}$ & \\
\hline Mutation (+) & 29 & 20 & 9 & & N/A & $\mathrm{N} / \mathrm{A}$ & \\
\hline
\end{tabular}

Table 1. Patients' clinicopathological characteristics according to NDRG2 and EGFR

\begin{tabular}{lccccc}
\hline Site & Alteration & Amino Acid Variation & NDRG2 (+) $(\mathrm{n}=20)$ & NDRG2 (-) $(\mathrm{n}=9)$ & Patients, $\mathrm{n}(\%)$ \\
\hline Exon 18 & Substitution & G719A & 0 & 1 & $1(3.4)$ \\
Exon19 & Deletion & E746-A750/ E746-T751/ L747-T751 & 10 & 5 & $15(51.7)$ \\
Exon 20 & Substitution & T790M & 1 & 0 & $1(3.4)$ \\
Exon 21 & Substitution & L858R & 9 & 3 & $12(41.4)$ \\
\hline
\end{tabular}


Table 2. EGFR mutations based on NDRG2 status

Table 3. Univariate Cox regression analysis for overall survival, disease-free survival and progression-free survival. 


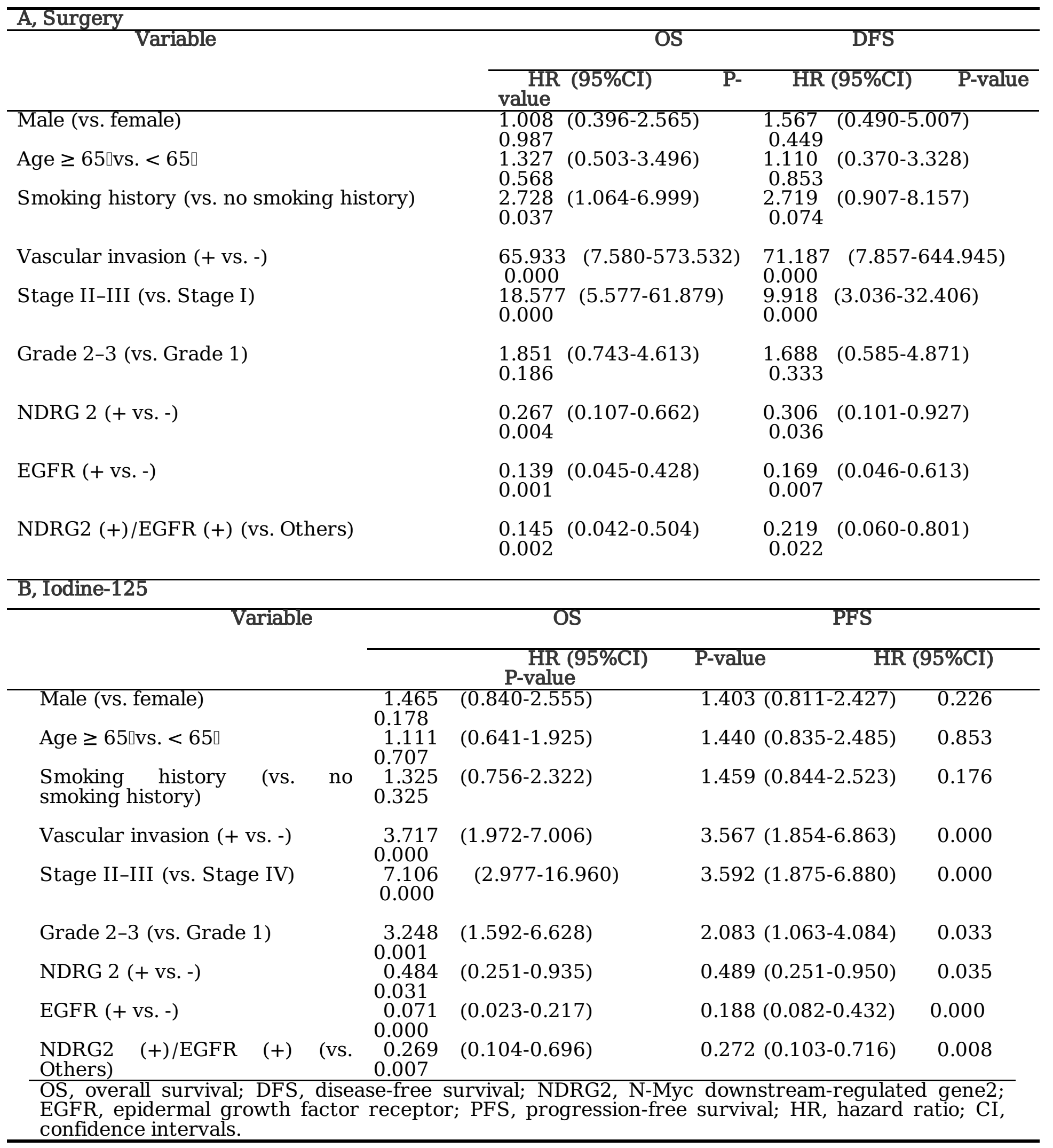

Table 4. Multivariate Cox regression analysis on overall survival, disease-free survival and progressionfree survival according to the groups based on the combination of NDRG2 and EGFR. 


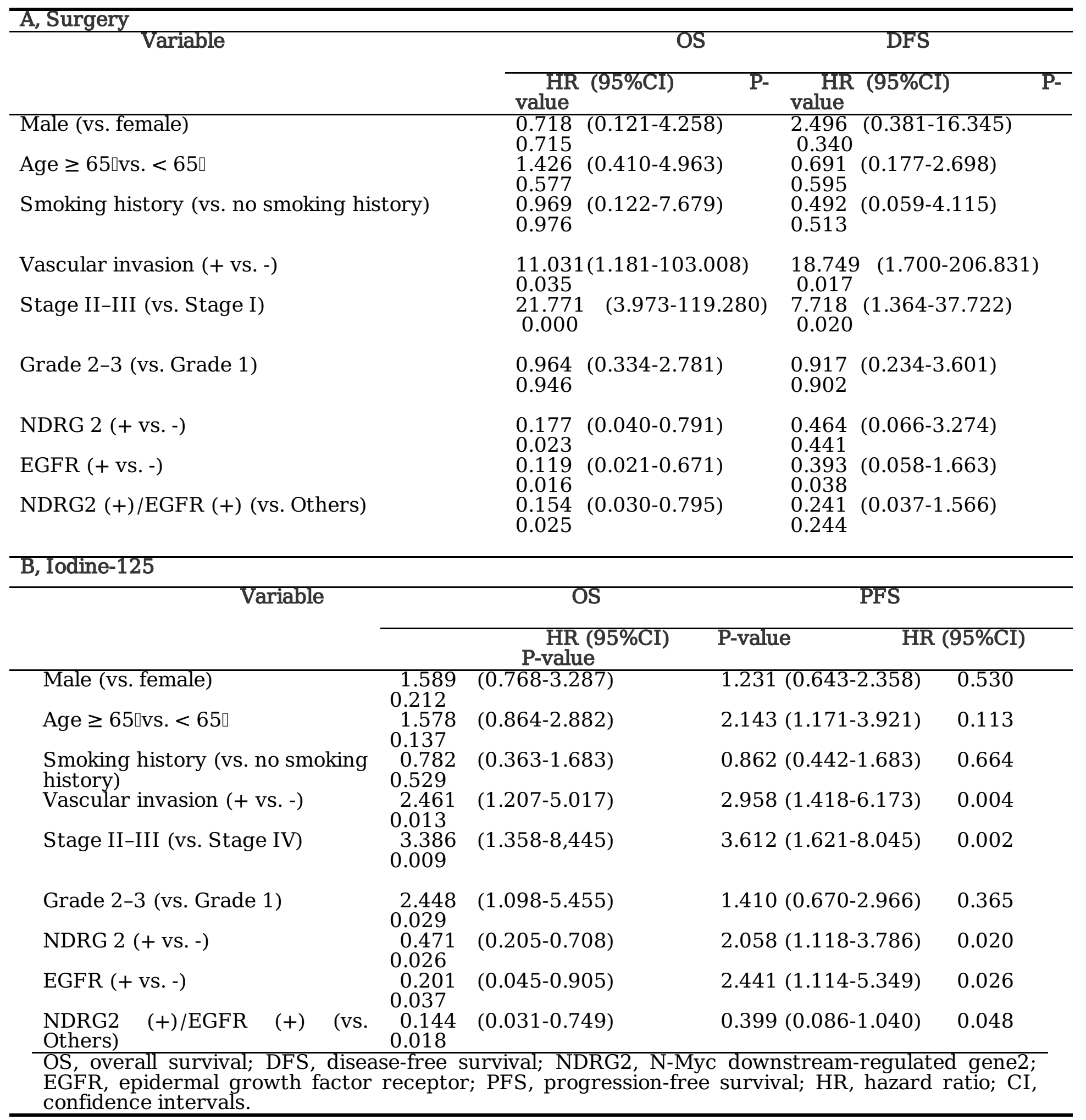

\section{Figures}




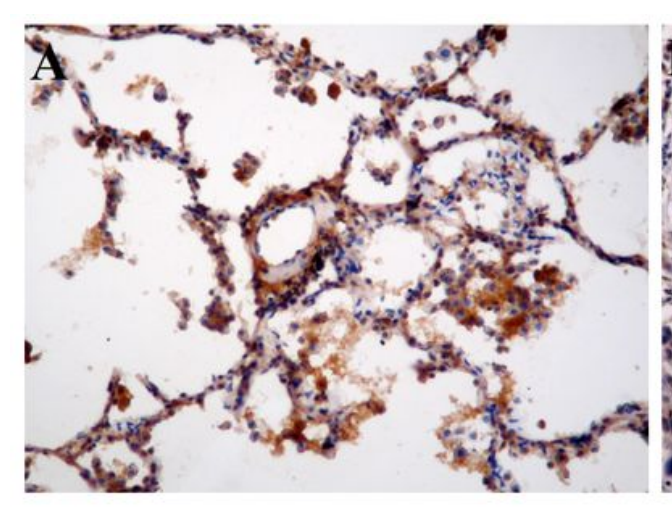

D

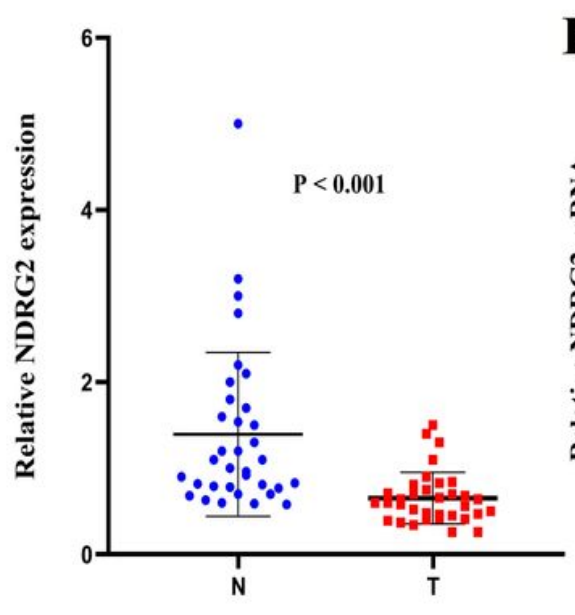

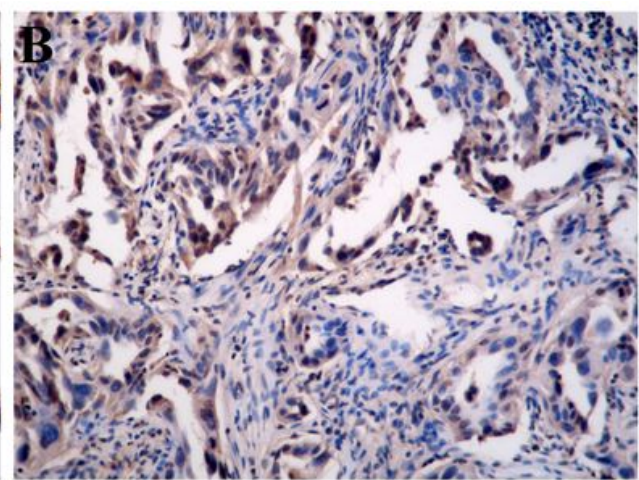

$\mathbf{E}$

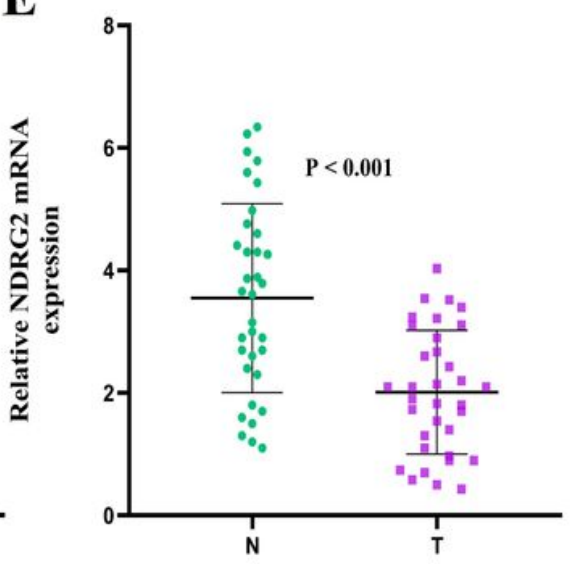

$\mathbf{F}$

G

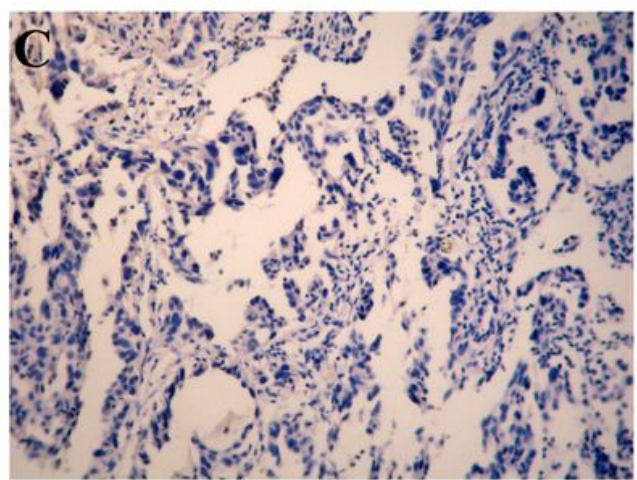

NDRG2
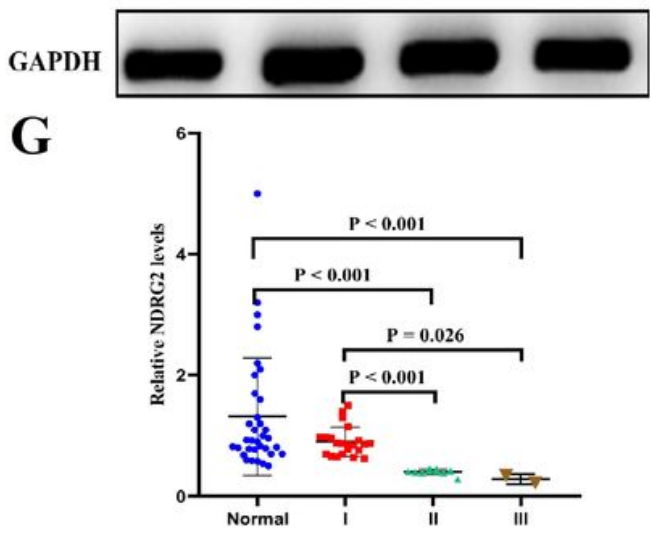

Figure 1

The expression level of NDRG2 in LUAD patients and in different stages. Immunohistochemistry showed the expression level of NDRG2 in adjacent normal tissues (Figure 1A), LUAD tissues (Figure 1B), and negative control (Figure 1C) (200× magnification). The expression level of NDRG2 was determined by western blot assay (Figure 1D) and qRT-PCR (Figure 1E). It was significantly downregulated in patients with LUAD tissues compared with that in adjacent normal tissues at both protein and mRNA levels. The expression of NDRG2 in different stages of LUAD (Figure 1F-G) $(p<0.001)$. Abbreviations: qRT-PCR, quantitative real-time reverse transcription polymerase chain reaction; GAPDH, glyceraldehyde-3phosphate dehydrogenase; T, LUAD tissues; $\mathrm{N}$, adjacent normal tissues. 


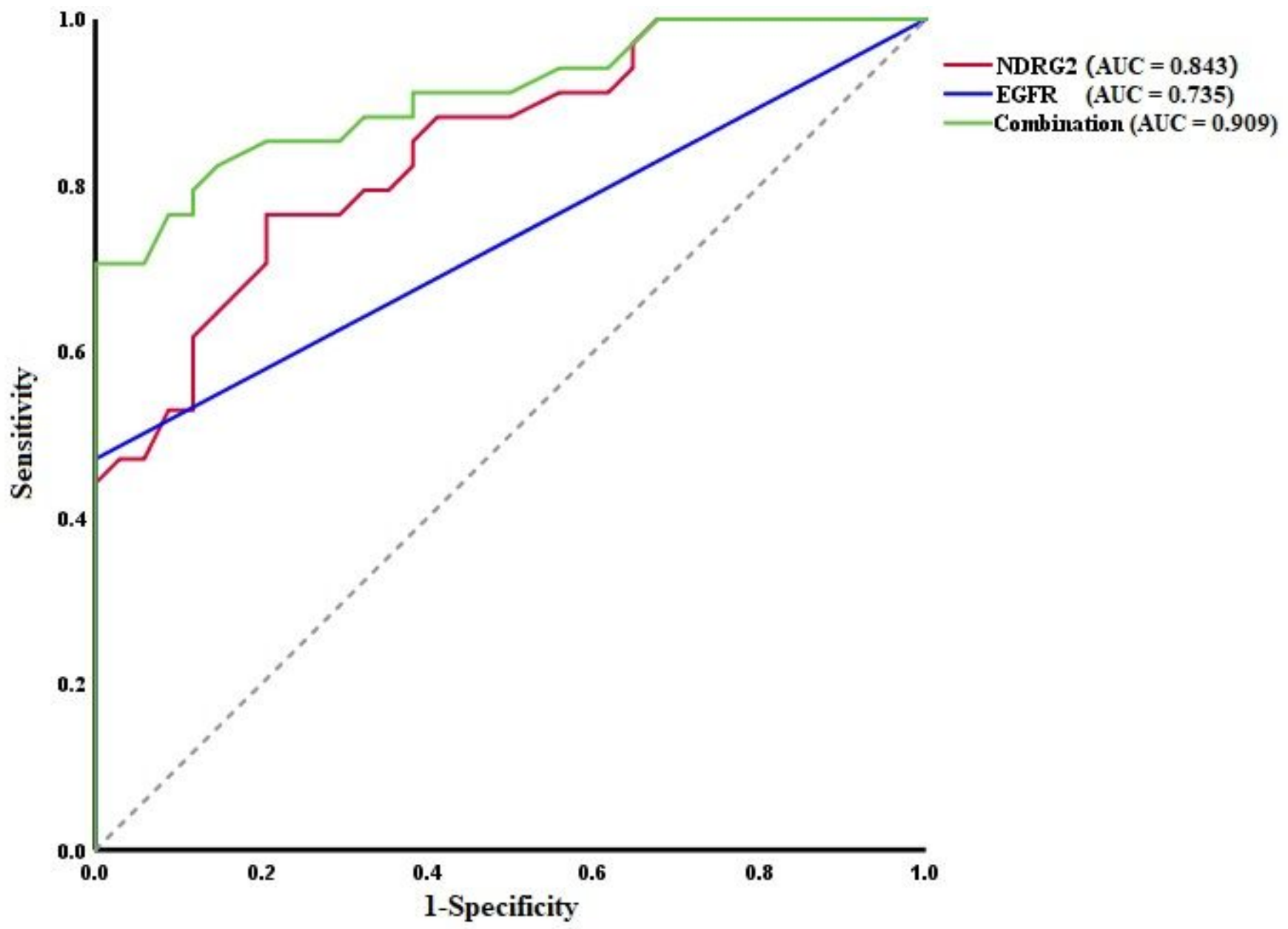

Figure 2

ROC curves of NDRG2, EGFR and the combination in LUAD vs. normal tissues. Abbreviations: ROC, receiver operating characteristic; AUC, area under curve. 
A

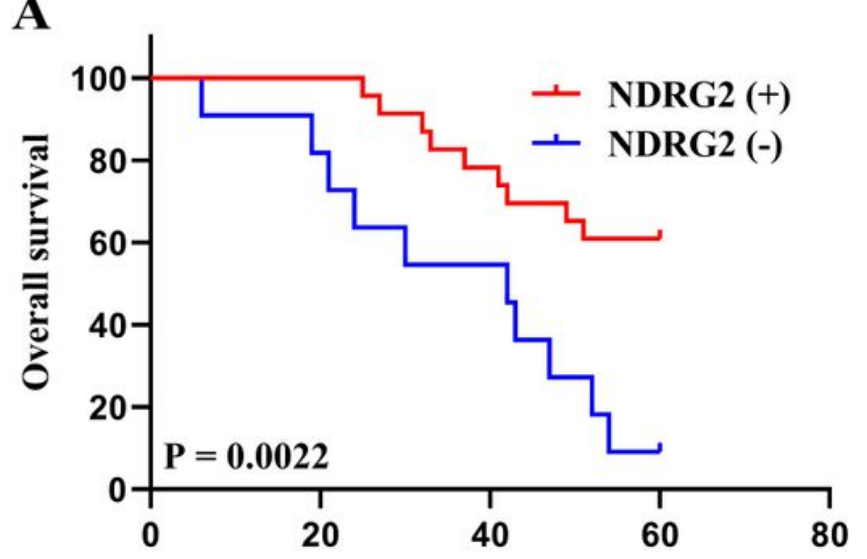

Time after surgery (months)

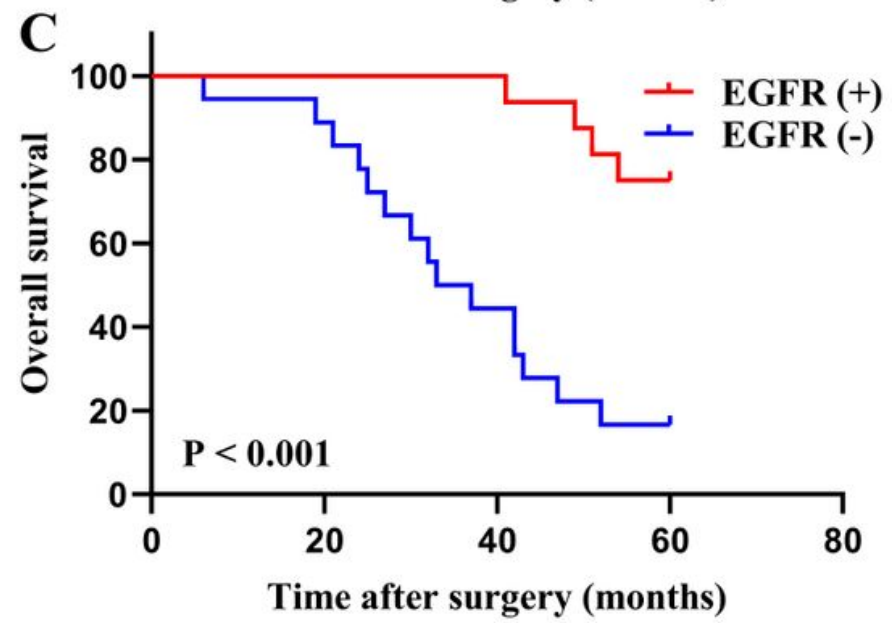

B

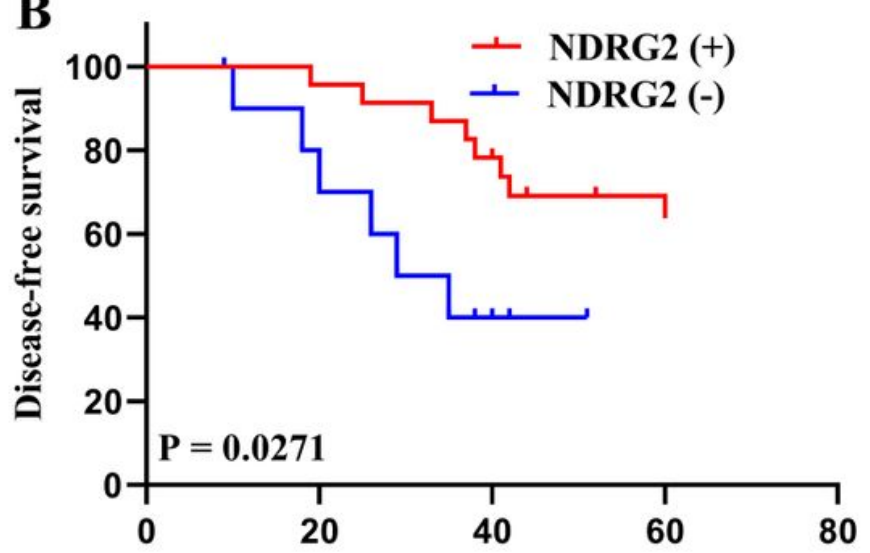

Time after surgery (months)

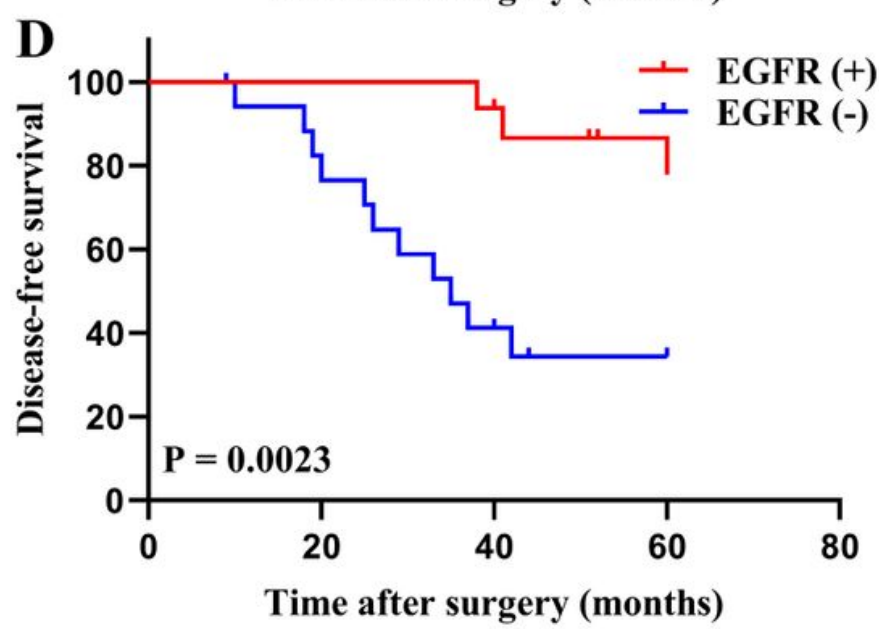

Figure 3

Kaplan-Meier curves for overall survival and disease-free survival according to NDRG2 expression and EGFR mutation status after surgery. (A) NDRG2 expression for overall survival. (B) NDRG2 expression for disease-free survival. (C) EGFR mutation status for overall survival. (D) EGFR mutation status for diseasefree survival. 


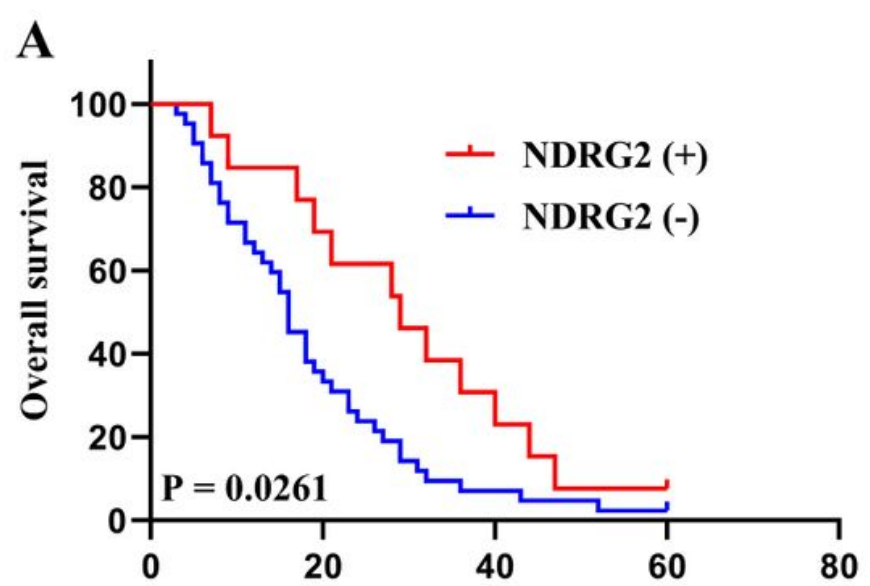

Time after iodine-125 implantation (months)

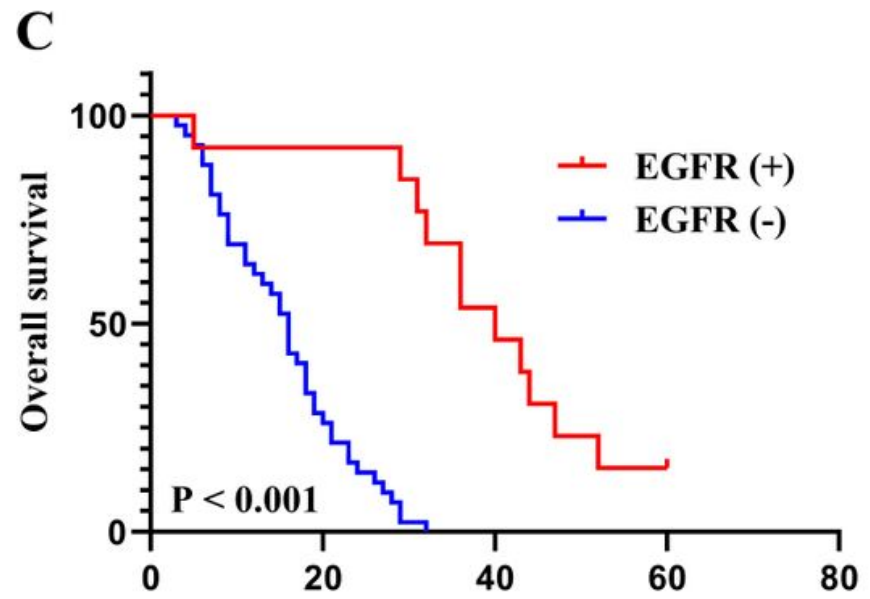

Time after iodine-125 implantation (months)

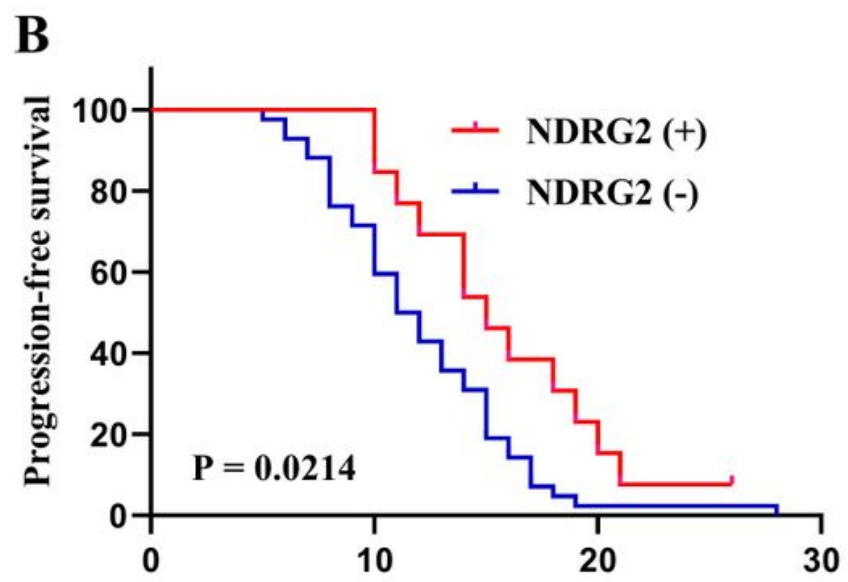

Time after iodine-125 implantation (months)

D

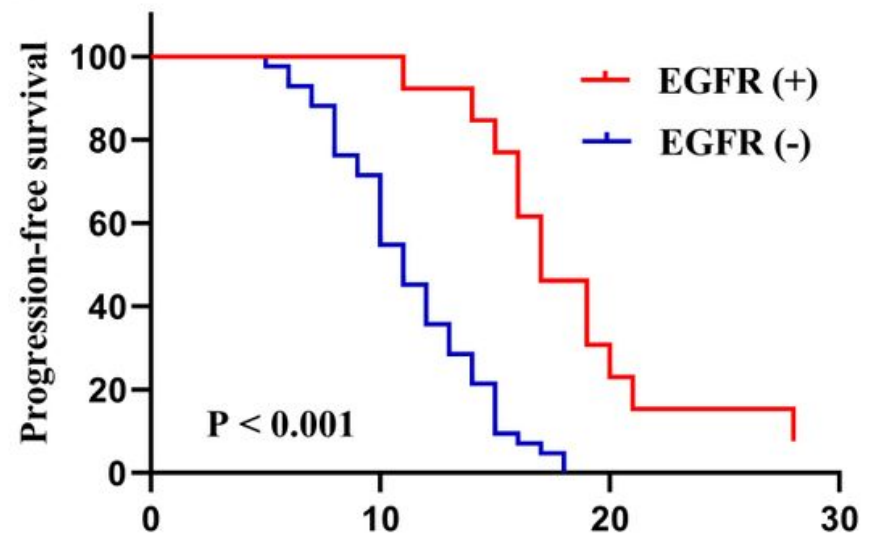

Time after iodine-125 implantation (months)

Figure 4

Kaplan-Meier curves for overall survival and progression-free survival according to NDRG2 expression and EGFR mutation status after iodine-125. (A) NDRG2 expression for overall survival. (B) NDRG2 expression for progression-free survival. (C) EGFR mutation status for overall survival. (D) EGFR mutation status for progression-free survival. 

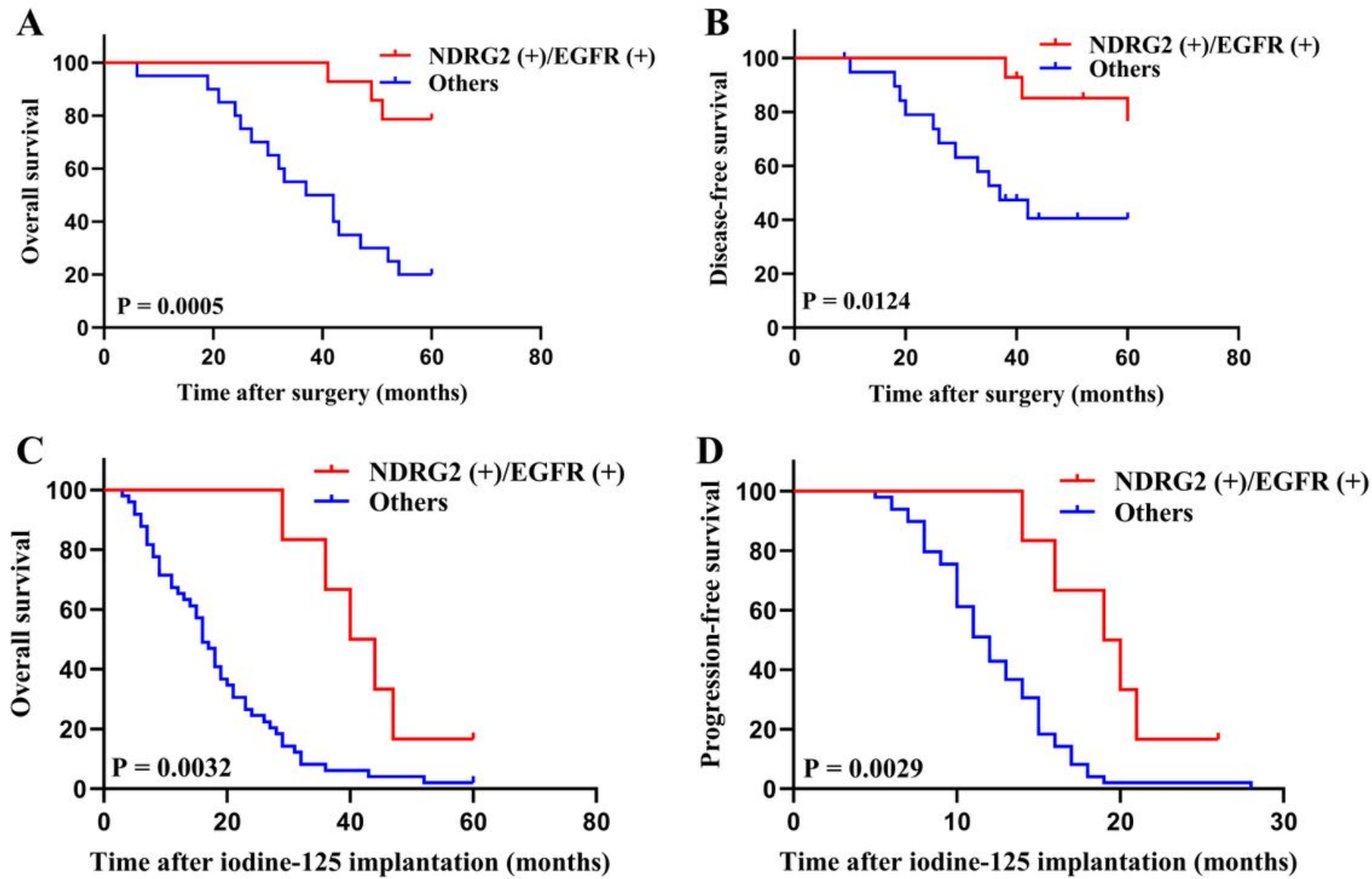

Figure 5

Kaplan-Meier curves for overall survival, disease-free survival and progression-free survival between NDRG2 (+)/EGFR (+) group and the others. (A, B) Overall survival and disease-free survival curves according to NDRG2 expression and EGFR mutation status in the LUAD patients after surgery. (C, D) Overall survival and progression-free survival curves according to NDRG2 expression and EGFR mutation status in the LUAD patients after iodine-125.

\section{Supplementary Files}

This is a list of supplementary files associated with this preprint. Click to download.

- originalSOCS1.jpg

- originaINDRG2.jpg

- originalCyclinD3GAPDH.jpg 